\section{El aprendizaje de habilidades de comunicación básicas en fisioterapia: una propuesta formativa utilizando el video $y$ el paciente simulado instructor}

\author{
Salvador Montull Morera \\ Data de defensa: $30 / 06 / 2015$
}

\begin{abstract}
Palabras clave: habilidades de comunicación, entrevista clínica, video, paciente simulado.
\end{abstract}

\title{
Resumen
}

Al observar, en los alumnos de la titulación de Fisioterapia de la Universidad Rovira i Virgili (URV), un posible déficit formativo en habilidades de comunicación (HC) para la realización de la entrevista clínica de Fisioterapia (ECF), se decide analizar el estado de la situación y aplicar una propuesta formativa (PF) que contribuya a mejorar el problema. La investigación se lleva a cabo en la Facultad de Medicina y Ciencias de la Salud (FMCS) de la URV, iniciándose en el curso académico 2007-8 y finalizando en el curso 2012-13. Participan como elementos integrantes de la muestra los alumnos del último curso de la Diplomatura de Fisioterapia, de cada curso académico, en los que se realizan las distintas intervenciones (Ciclo I, Fase diagnóstica; Ciclo I, Propuesta formativa I; Ciclo II, Propuesta formativa II).

El método de investigación utilizado es mixto, cualitativo y cuantitativo, aunque el acercamiento a la realidad es eminentemente cualitativo y se basa en el método de investigación - acción (I-A). La perspectiva cualitativa es a su vez sociocrítica, pues se busca interpretar la realidad y proponer cambios dirigidos a modificar determinados aspectos de la misma a partir de una acción concreta. Además se utilizan métodos descriptivos y de comparación entre grupos con la finalidad de refinar las sucesivas propuestas formativas. Se toma en consideración la información procedente de: los alumnos, de los pacientes simulados, del investigador, del grupo de trabajo y de dos observadores externos. Los métodos de recogida de la información se basaron en: la cumplimentación de encuestas semiestructuradas (profesores y alumnos), las videograbaciones (seminarios, entrevistas, sesiones de coordinación, etc.), el diario del investigador, la Escala MAPE 2 (mapa de frases clave para pacientes estandarizados) y el Cuestionario CICAA (conectar, identificar, comprender, acordar y ayudar). La

\footnotetext{
a Universitat Rovira i Virgili
} 
información cualitativa se categorizó y se analizó mediante el programa weft/ QDA (versión 1.0.1). Para el análisis de la información cuantitativa se utilizó el paquete estadístico SPSS (versión 19.0). Para determinar la normalidad de las variables se utilizó el estadístico Kolmogorov-Smirnof. Las variables categóricas se expresaron en porcentajes y las cuantitativas mediante medidas de tendencia central y de dispersión. Para analizar los efectos de las propuestas formativas realizadas se realizaron pruebas de contraste de hipótesis para las variables cuantitativas y cualitativas, aceptándose un error tipo I (5\%) para el contraste lateral de hipótesis en relación a los análisis realizados. Finalmente se trianguló la información.

Se diseñan 2 Ciclos de I-A. La primera parte del Ciclo I se dedica a analizar y a definir el diagnóstico de la situación (Fase diagnóstica) y se realiza durante los cursos académicos 2007-08 y 2008-2009. Durante la segunda parte del Ciclo I (Curso académico 2009-10) se aplica la Propuesta formativa I (PF I). Durante el Ciclo II (201011 a 2013) se introducen modificaciones que darán lugar a la aplicación de la $P$. formativa II (PF II) y a la concreción de la P. formativa final (PFF).

Mientras se aplican las distintas propuestas formativas de cada ciclo, los alumnos participarán en una estrategia didáctica basada en el estudio de casos (estudio de descripciones, resolución de problemas y desempeño y resolución de situaciones de simulación). Los alumnos se dividen, de forma aleatoria, en dos grupos: B (Grupo de intervención) y A (Grupo control) durante la aplicación de cada PF realizan un método distinto de aprendizaje combinado (B-learning) siendo su evaluación formativa y sumativa. Durante el Ciclo I (Fase diagnóstica) los alumnos cumplimentaron encuestas semiestructuradas, entrevistaron a un PSE y fueron entrevistados por el investigador. La información obtenida permitió iniciar la $2^{a}$ parte del Ciclo I con la aplicación de la PF I. En este caso los alumnos del GI realizaron las siguientes actividades (tiempo de dedicación: 24 horas): 2 seminarios (clase magistral, debates, construcción del concepto y de las fases de la entrevista clínica, identificación de los errores habituales), 6 E-actividades (descripción de los objetivos y de los errores de cada fase de la ECF, análisis de videos de entrevistas y resolución de casos), 3 entrevistas a 3 PSE diferentes, visualización del video y retroacción. Los alumnos del GC realizaron (tiempo de dedicación 14 horas): 4 clases magistrales, 3 E-actividades (revisión conceptual de las fases de la ECF), 1 entrevista a 1 PSE y visualización del video de la entrevista realizada y retroacción. Durante el Ciclo II los alumnos del GI participan realizan (con una dedicación de 25 horas): 3 seminarios presenciales (análisis de video clips y 2 sesiones de Role play), 3 E-actividades (resolución de casos, análisis de video clips), 3 entrevistas a pacientes simulados estandarizados distintos (obteniendo la retroacción del tercer PSE) y 3 sesiones de visualización de los videos de las entrevistas en grupo (correspondientes a cada una de las entrevistas realizadas), donde obtienen retroacción de los compañeros (con el apoyo de la Guía Calgary Cambridge) y del profesor. Los alumnos del GC realizan (con un tiempo de dedicación de 16 h): 3 clases magistrales, $3 \mathrm{E}$-actividades (resolución de preguntas conceptuales), una entrevista al PSE y observan el video de su entrevista y obtienen retroacción. 
Expondremos las conclusiones teniendo en cuenta: el análisis diagnóstico, las propuestas formativas realizadas y el potencial del video y del PSE. En relación al análisis diagnóstico (Ciclo I, Fase diagnóstica): los alumnos muestran una gran inseguridad a la hora de realizar entrevistas clínicas y un déficit formativo en HC para realizar la ECF. La fase de la entrevista en la que mostraron más inseguridad fue la de explicación y planificación. Los alumnos solo realizan entrevistas clínicas de forma excepcional durante el periodo de prácticas. Los alumnos pierden información relevante, durante la fase de recogida de la información del paciente, por un déficit de habilidades comunicativas durante el encuentro alumno-paciente. Los contenidos del currículo relacionados con las $\mathrm{HC}$ son insuficientes y no se aplican de forma coordinada entre la teoría y la práctica clínica. En relación a las propuestas formativas realizadas: el método de aprendizaje mezclado (B-learning) puede facilitar el aprendizaje de HC para realizar la ECF. Los métodos que más influyeron, según los alumnos del GI, en su formación fueron (en orden decreciente): la realización de entrevistas con cada PSE, la obtención de retroacción por parte del PSE, la observación del vídeo de las entrevistas realizadas, los seminarios, las E-actividades y el método role play. Tras la aplicación de las propuestas formativas I y II, los alumnos mejoraron sus HC para realizar la EC. Existe una dificultad, por parte de los alumnos, para integrar el proceso de recogida de la información del paciente con el proceso de razonamiento clínico. Las fases de la ECF donde los alumnos (del GI) mostraron mayores dificultades fueron por este orden: la fase de recogida de la información, la obtención del diagnóstico de Fisioterapia y la explicación del problema al paciente. En relación al potencial del vídeo y del paciente simulado: EI PSE puede jugar a la vez el rol de paciente y de instructor mientras proporciona retroacción al alumno en un medio controlado. La observación del vídeo de las entrevistas clínicas permite analizar el manejo de las HC y aprovechar la retroacción proporcionada por los mismos alumnos y por el profesor. La Guía Calgary Cambridge ayuda a optimizar las sesiones de retroacción mientras se visualizan las videograbaciones de las entrevistas. 